OPEN ACCESS

Edited by:

Walter Gottlieb Land, Université de Strasbourg, France

Reviewed by: Seigo Okada, Yamaguchi University, Japan Zhengkai Wei,

Foshan University, China

*Correspondence: Kentaro Ueno ukenta@m3.kufm.kagoshima-u.ac.jp

Specialty section:

This article was submitted to Molecular Innate Immunity, a section of the journal

Frontiers in Immunology

Received: 11 December 2020 Accepted: 04 May 2021 Published: 17 May 2021

Citation:

Ueno $K$, Nomura $Y$, Morita $Y$ and Kawano $Y$ (2021) Prednisolone Suppresses the Extracellular Release of HMGB-1 and Associated Inflammatory Pathways in Kawasaki Disease.

Front. Immunol. 12:640315. doi: 10.3389/fimmu.2021.640315

\section{Prednisolone Suppresses the Extracellular Release of HMGB-1 and Associated Inflammatory Pathways in Kawasaki Disease}

\author{
Kentaro Ueno ${ }^{1 *}$, Yuichi Nomura ${ }^{2}$, Yasuko Morita ${ }^{1}$ and Yoshifumi Kawano ${ }^{1}$ \\ ${ }^{1}$ Department of Pediatrics, Kagoshima University Graduate School of Medical and Dental Sciences, Kagoshima, Japan, \\ ${ }^{2}$ Department of Pediatrics, Kagoshima City Hospital, Kagoshima, Japan
}

Innate immune activity plays an essential role in the development of Kawasaki disease (KD) vasculitis. Extracellular release of high mobility group box-1 (HMGB-1), an endogenous damage-associated molecular pattern protein that can activate the innate immune system and drive host inflammatory responses, may contribute to the development of coronary artery abnormalities in KD. Prednisolone (PSL) added to intravenous immunoglobulin treatment for acute KD may reduce such abnormalities. Here, we evaluate the dynamics of HMGB-1 and therapeutic effects of PSL on HMGB-1-mediated inflammatory pathways on $K D$ vasculitis in vitro. Serum samples were collected prior to initial treatment from patients with $K D$, systemic juvenile idiopathic arthritis (SJIA), and from healthy controls $(V H)$, then incubated with human coronary artery endothelial cells (HCAECs). Following treatment of KD serum-activated HCAECs with PSL or PBS as a control, effects on the HMGB-1 signaling pathway were evaluated. Compared to that from VH and SJIA, KD serum activation induced HCAEC cytotoxicity and triggered extracellular release of HMGB-1. KD serum-activated HCAECs up-regulated extracellular signal-regulated kinase (ERK)1/2, cJun N-terminal kinase (JNK) and, p38 phosphorylation in the cytoplasm and nuclear factor

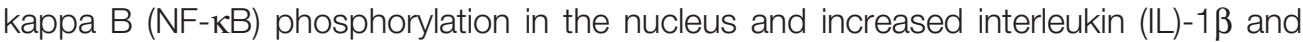
tumor necrosis factor (TNF)- $\alpha$ production. PSL treatment of KD serum-activated HCAECs inhibited extracellular release of HMGB-1, down-regulated ERK1/2, JNK, p38, and NF-KB signaling pathways, and decreased IL-1 $\beta$ and TNF- $\alpha$ production. Our findings suggest that extracellular HMGB-1 plays an important role in mediating KD pathogenesis and that PSL treatment during the acute phase of KD may ameliorate HMGB-1-mediated inflammatory responses in KD vasculitis.

Keywords: pediatrics, Kawasaki disease (KD), DAMPs (damage-associated molecular patterns), prednisolone, high mobility group box-1 


\section{INTRODUCTION}

Kawasaki disease (KD), an acute systemic vasculitis of unknown etiology, commonly occurs in children (1), and can ultimately lead to complex coronary artery abnormalities (CAAs). Despite standard treatment with high-dose intravenous immunoglobulin (IVIG) and aspirin, approximately $10 \%$ to $20 \%$ of patients experience persistent or recurrent fever and appear to have elevated risk of developing CAAs $(2,3)$. Notably, combined treatment with prednisolone (PSL) is more effective in preventing CAAs than IVIG alone in non-responders, thereby reducing the need for additional rescue treatments (4). Thus, corticosteroid combination therapy is considered a promising pre-emptive primary treatment $(5,6)$.

Innate immune activity is integral toward $\mathrm{KD}$ vasculitis etiology (7). High mobility group box-1 (HMGB-1), a representative damage-associated molecular pattern (DAMP) protein, plays a central role in regulating programmed cell death and survival (8). As sentinel innate immune cells, endothelial cells release DAMPs as endogenous danger signals that alert the innate immune system to unscheduled cell death, microbial invasion, and stress (9). Extracellular HMGB-1 coordinates cellular responses associated with immune system activation, cell migration, cell growth, and tissue repair and regeneration, and binds to receptors, such as receptor for advanced glycation end products (RAGE) and Toll-like receptors (TLRs) to activate proinflammatory responses. Downstream signaling involving mitogen-activated protein kinase (MAPK) such as extracellular signal-related kinase (ERK)/c-Jun $\mathrm{N}$-terminal kinase (JNK)/p38 and nuclear factor kappaB $(\mathrm{NF}-\kappa \mathrm{B})$ facilitates cellular responses including inflammatory cytokine, chemokine, and corresponding receptor expression (10). Injury-evoked increased HMGB-1mediated inflammatory responses can increase cardiovascular disease severity (11-14). Notably, serum HMGB-1 and S100 protein levels are also elevated in patients in the acute phase of KD (15-17). Moreover, Nucleotide-binding oligomerization domain-like receptor family, pyrin domain-containing 3 (NLRP3)-dependent endothelial cell pyroptosis via HMGB-1/ RAGE/cathepsin B signaling may contribute to coronary artery endothelial cell (CAEC) damage in KD vasculitis (18). Thus, DAMP-mediated innate immune system activation may facilitate pathological inflammatory responses in KD vasculitis.

We hypothesize that PSL treatment, a standard antiinflammatory therapy, may suppress inflammation in $\mathrm{KD}$ by reducing inflammatory cytokines and DAMPs produced by

\footnotetext{
Abbreviations: KD, Kawasaki disease; HMGB-1, high mobility group box-1; PSL, Prednisolone; sJIA, systemic juvenile idiopathic arthritis; HCAECs, human coronary artery endothelial cells; ERK, extracellular signal-regulated kinase; JNK, c-Jun N-terminal kinase; NF- $\kappa$ B, nuclear factor kappa B; NLRP3, Nucleotide-binding oligomerization domain-like receptor family, pyrin domaincontaining 3; IL, interleukin; TNF, tumor necrosis factor; CAA, coronary artery abnormalities; IVIG, intravenous immunoglobulin; DAMPs, damage-associated molecular pattern; RAGE, receptor for advanced glycation end products; TLRs, Toll-like receptors; PBS, phosphate buffered saline; MTT, 3-(4,5-dimethylthiazol2-yl)-2,5-diphenyl tetrazolium bromide; GAPDH, glyceraldehyde-3-phosphate dehydrogenase; RT-PCR, reverse transcription-polymerase chain reaction.
}

CAECs consequent to acute $\mathrm{KD}$ vasculitis. In this study, we clarified the role of HMGB-1 and evaluated the in vitro therapeutic effects of PSL on HMGB-1-mediated inflammatory responses in CAECs during acute $\mathrm{KD}$ vasculitis.

\section{MATERIALS AND METHODS}

\section{Patients}

The study was approved by the Kagoshima University and Kagoshima City Hospital Ethics Committee and performed in accordance with the International Conference on Harmonization guidelines for Good Clinical Practice and the Declaration of Helsinki (approval number: MD26-156, Approval date: January 13, 2016). We enrolled eight consecutive patients undergoing treatment for $\mathrm{KD}$ at the host institution applying the following exclusion criteria: 1) cardiovascular disease, hematological disease, congenital malformations, primary disease of major organs, and genetic/chromosomal abnormalities; 2) bacteremia or sepsis with positive blood culture; 3 ) recurrent KD symptoms; 4) previous use of corticosteroids or immunosuppressive treatment; 5) development of coronary artery lesions. We used four healthy subjects and four patients with systemic juvenile idiopathic arthritis (sJIA) as vehicle and disease controls, respectively. Written informed consent was obtained from the parents of all study participants and serum samples were collected from the patients and healthy subjects.

$\mathrm{KD}$ was defined using the Japanese criteria (19). The first day of illness was defined as the first day of fever. Treatment was initiated when $\mathrm{KD}$ was considered highly likely even if all $\mathrm{KD}$ criteria were not met. Patients with KD received a single IVIG infusion $(2 \mathrm{~g} / \mathrm{kg})$ together with aspirin $(30 \mathrm{mg} / \mathrm{kg} / \mathrm{day}$, decreased to 3 to $5 \mathrm{mg} / \mathrm{kg} /$ day if afebrile for $\geq 28$ days following fever onset).

\section{Blood-Sample Collection}

Serum samples were collected from patients with $\mathrm{KD}$ and sJIA before initial treatment and from healthy controls, separated by centrifugation $(700 \times g, 15 \mathrm{~min})$, and stored at $-40^{\circ} \mathrm{C}$.

\section{Endothelial Cell Culture and Preparation}

Primary human CAECs (HCAECs) were purchased from PromoCell (Heidelberg, Germany) and cultured using MV 2 kit endothelial cell growth medium (PromoCell). Medium was changed every $24 \mathrm{~h}$. Cells at $70 \%$ to $80 \%$ confluence were seeded into 96-, 8-, or 6-well microplates for assays and fluorescence microscopy. Third passage HCAECs were used for experiments. Vehicle (VH) and KD controls comprised HCAECs at $90 \%$ confluence incubated for $24 \mathrm{~h}$ in MV 2 basal medium (PromoCell) with 7.5\% healthy volunteer or KD patient serum, respectively. Serum-activated HCAECs treated with phosphate buffered saline (PBS) and PSL $\left(10^{-6} \mathrm{M} /\right.$ well (20); Shionogi, Osaka, Japan) for $24 \mathrm{~h}$ were defined as $\mathrm{KD}+\mathrm{PBS}$ and $\mathrm{KD}+$ PSL HCAECs, respectively. After each experiment, media were replaced with serum-free fresh MV 2 growth media to discriminate serum cytokine effects. ELISA evaluation of final washes demonstrated TNF- $\alpha$ levels (R\&D systems, Minneapolis, MN, 
USA) below detectable limits $(<5.5 \mathrm{pg} / \mathrm{mL}$ ) (Supplementary Figure 1). Experiments were repeated at least twice and media were maintained between $\mathrm{pH} 7.2$ and 7.4.

\section{Analysis of Serum-Activated HCAEC Viability and Cytotoxicity}

Cell viability was measured using 3-(4,5-dimethylthiazol-2-yl)-2,5diphenyl tetrazolium bromide (MTT) assay (Dojindo, Kumamoto, Japan). HCAECs were cultured in growth medium in 96-well plates $\left(0.5 \times 10^{4}\right.$ cells/well $)$. Following each experiment, medium was replaced with fresh MV 2 basal medium and the final sample volume adjusted to $100 \mu \mathrm{L} /$ well. Samples were subjected to MTT assay according to manufacturer instructions and measured in duplicate, using Microplate Reader (Tecan Infinite M200).

Cytotoxicity was evaluated via fluorescence to measure the activity of dead-cell protease, which is released from cells with impaired membrane integrity, using a CytoTox-Glo cytotoxicity assay (Promega, Madison, WI, USA) according to manufacturer instructions. Briefly, HCAECs $\left(0.5 \times 10^{4}\right.$ cells/well $)$ were cultured in growth medium in 96-well plates. After each experiment, medium was replaced with fresh MV 2 basal medium $(100 \mu \mathrm{L} /$ well final volume). Fluorescence measured using a Tristar multimode microplate reader (LB 941; Berthold Technologies, Oak Ridge, TN, USA), was directly proportional to the number of dead cells. Each sample was measured in duplicate.

\section{Assessment of Extracellular HMGB-1 Released From KD Serum-Activated HCAECs}

HMGB-1 content in supernatant released from KD serumactivated HCAECs for $24 \mathrm{~h}$ was measured in duplicate using a commercial ELISA kit (Shino-Test Corporation, Tokyo, Japan) according to manufacturer instructions. The minimum HMGB-1 detection value was $1.0 \mathrm{ng} / \mathrm{mL}$.

\section{Quantitative Analysis of Receptors on KD Serum-Activated HCAECs}

Total RNA samples were extracted from cell lysates of serumactivated HCAECs using the RNeasy Mini Kit (\#74104; QIAGEN, Hilden, Germany) according to manufacturer's instructions. Reverse transcription was performed using a PrimeScript RT Reagent Kit (TaKaRa, Tokyo, Japan) according to manufacturer's instructions. An equivalent volume of cDNA solution was used for real-time PCR quantification using a Thermal Cycler Dice Real Time System (TaKaRa), with glyceraldehyde-3-phosphate dehydrogenase $(G A P D H)$ as the internal standard. At least two biological replicates were performed and specific PCR product amplification was confirmed by melting curve analysis. Gene expression was calculated using the $2^{-\triangle \Lambda C T}$ method. Table 1 lists primer sequences (GAPDH, RAGE, TLR2, and TLR4) and RT-PCR conditions.

\section{Soluble RAGE (sRAGE) Production}

Supernatant sRAGE levels released from KD serum-activated HCAECs for $24 \mathrm{~h}$ were measured in duplicate using a commercially available ELISA kit (R\&D Systems) according to manufacturer instruction. The minimum sRAGE detection value was $4.21 \mathrm{pg} / \mathrm{mL}$.

\section{Immunofluorescence Staining for HMGB-1}

HCAECs prepared on 8-well imaging chamber $\left(7.0 \times 10^{4}\right.$ cells/well $)$ were incubated with MV 2 growth medium $\left(37^{\circ} \mathrm{C}, 5 \% \mathrm{CO}_{2}\right) . \mathrm{VH}$, $\mathrm{KD}$ control, $\mathrm{KD}+\mathrm{PBS}$, and $\mathrm{KD}+\mathrm{PSL}$ HCAECs were washed with PBS, fixed, permeabilized using the Image-iT fixation/ permeabilization kit (Invitrogen, Grand Island, NY, USA) and intracellularly stained with Alexa Fluor 594 anti-HMGB-1 (red) in blocking buffer overnight at $4^{\circ} \mathrm{C}$. Nuclei were counterstained using ProLong Gold Antifade reagent with 4',6-diamidino-2phenylindole (DAPI, blue) (Life Technologies, Eugene, OR, USA). Randomly selected cells $(n=100)$ from each group were observed using fluorescence microscopy (Keyence BZ-X700; Carl Zeiss, Oberkochen, Germany). Quantitative analysis of immunofluorescence staining was performed using ImageJ software (National Institutes of Health, Bethesda, MD, USA) (21).

\section{Western Blot Analysis}

Third-passage HCAECs were cultured in 6-well plates $\left(4.0 \times 10^{5}\right.$ cells/ well) with growth medium. After each experiment, adherent cells were lysed using the total protein extraction kit for animal cultured cells and tissues (Invent Biotechnologies, Plymouth, MN, USA) and prepared for immunoblotting. Each $15 \mu \mathrm{L}$ sample was subjected to $10 \%$ gradient SDS-PAGE and electrotransferred onto a polyvinylidene difluoride membrane, then immunoblotted using primary antibodies against phosphorylated ERK 1/2 (p-ERK; Cell Signaling Technology (CST), Beverly, MA, USA; 1:2,000 dilution), ERK 1/2 (CST; 1:1,000), phosphorylated stress-activated protein kinase (SAPK)/c-Jun amino terminal kinase (JNK) (CST; 1:1,000), SAPK/JNK (CST; 1:1,000), phosphorylated p38 (CST; 1:1,000), p38 (CST; 1:1,000), anti-NLRP3 (CST; 1:1,000), anti-cleaved Caspase-1, (CST; 1:1,000), IL-1 $\beta$ (CST; 1:1,000), and TNF- $\alpha$ (CST; 1:1,000), followed by horseradish peroxidase-conjugated secondary antibody (Medical \& Biological Laboratories, Nagoya, Japan; 1:1,000). For NF$\kappa \mathrm{B}$ p65, nuclear and cytoplasmic proteins were extracted using an extraction kit (SC-003, Invent Biotechnologies) according to manufacturer instructions. Each $15 \mu \mathrm{L}$ sample was subjected to $10 \%$ gradient SDS-PAGE and electrotransferred onto a polyvinylidene difluoride membrane and immunoblotted using primary antibodies against phosphorylated NF- $\kappa B$ p 65 (p-NF- $\kappa B$ p65; CST; 1:1,000) or NF- $\mathrm{B}$ p65 (CST; 1:1,000), followed by horseradish peroxidase-conjugated secondary antibody (Medical \& Biological Laboratories; 1:1,000). Housekeeping protein, such as $\beta$-actin and glyceraldehyde-3-phosphate dehydrogenase (GAPDH) were used as loading controls on the account of their expression levels. Proteins were visualized by chemiluminescence with SignalFire ECL Reagent (CST), and quantified using Fluor Chem FC2 (Alpha Innotech Kasendorf, Germany). All blotting experiments were repeated at least twice.

\section{Statistical Analysis}

Continuous variables are reported as median values with interquartile ranges (IQR; 25th-75th percentiles). Categorical variables are presented as frequencies and percentages. Baseline comparisons between patients were performed using Student's t-tests, Mann-Whitney $U$-tests, or $\chi^{2}$ analysis (with Yates' correlation or Fisher's exact test, as appropriate). Differences between $>2$ groups were evaluated by one-way ANOVA followed 
TABLE 1 | Primer sequences and PCR conditions.

\begin{tabular}{|c|c|c|c|c|}
\hline mRNA & Primer sequences & Annealing time and temperature $\left({ }^{\circ} \mathrm{C}\right)$ & Cycle no. & Fragment length/base pairs \\
\hline TLR2 & $\begin{array}{l}\text { sense: 5'-GGCTTCTCTGTCTTGTGACC-3' } \\
\text { antisense: 5'-GGGCTTGAACCAGGAAGACG-3' }\end{array}$ & $0.5 \mathrm{~min} ; 49$ & 32 & 294 \\
\hline TLR4 & $\begin{array}{l}\text { sense: 5'-TTGTATTCAAGGTCTGGCTGG-3' } \\
\text { antisense: 5'-GCAAACCTTGAAACTCAAGCC-3' }\end{array}$ & $0.5 \mathrm{~min} ; 47$ & 32 & 438 \\
\hline
\end{tabular}

GAPDH, Glyceraldehyde-3-phosphate dehydrogenase; RAGE, receptor for advanced glycation end products; TLR2, Toll-like receptor 2; TLR4, Toll-like receptor 4. Bold values indicate statistically significance in patient characteristics between KD group and sJIA group.

by the Bonferroni or Games-Howell test and the Kruskal-Wallis test with the Dunn's post hoc test. The former was performed when the variables showed a normal distribution; otherwise, the latter was used. All statistical analyses were performed using SPSS statistical software (v.25.0; SPSS Japan Inc., Tokyo, Japan). A two-tailed $P<0.05$ was considered statistically significant.

\section{RESULTS}

\section{Clinical Characteristics and Laboratory Findings in Patients With KD and sJIA}

Sera from eight patients with KD (median, 1.6 years) and four with sJIA (median 7.8 years) were evaluated. Table 2 lists patient clinical characteristics and laboratory findings. Age and body weight significantly differed between the groups as KD and sJIA patients exhibited different peak ages of onset. Laboratory data, including baseline white blood cell counts, neutrophil counts, and C-reactive protein levels, did not significantly differ with the exception of total protein and sodium levels.

\section{Serum Concentration From Patients With KD Necessary to Serum-Activate HCAECs}

To determine the serum concentration from patients with $\mathrm{KD}$ necessary to exert cytotoxic effects on HCAECs, we first exposed HCAECs to four independent $\mathrm{KD}$ sera concentrations, $0 \%$ (untreated), 5\%, 7.5\%, and $12.5 \%$, for $24 \mathrm{~h}$. KD serum induced significant cytotoxic effects on HCAECs at concentrations $\geq 7.5 \%$ (Supplementary Figure 2A) while maintaining cellular viability (Supplementary Figure 2B). Therefore, $7.5 \% \mathrm{KD}$ serum was used for subsequent in vitro experiments.

\section{Proliferative Activity and Cytotoxicity of Serum-Activated HCAECs}

MTT assay revealed that cell proliferation of serum-activated HCAECs from KD controls was significantly higher than that from the $\mathrm{VH}$ and sJIA groups (each $P<0.001$ ) (Figure 1A). In $\mathrm{KD}$, the proliferation of serum-activated HCAECs from the KD+PSL group was significantly lower than those of serum-activated $\mathrm{KD}$ controls and $\mathrm{KD}+\mathrm{PBS}$ group $(P<0.002$, and $P=0.012$, respectively) (Figure 1B). Cytotoxicity in serum-activated HCAECs from KD controls was significantly higher than those in the $\mathrm{VH}(P=0.007)$ and sJIA groups $(P=0.044)$ (Figure $1 C)$. In $\mathrm{KD}$, cytotoxicity of serumactivated HCAECs from the KD+PSL group tended to be lower than those of serum-activated $\mathrm{KD}$ controls and $\mathrm{KD}+\mathrm{PBS}$ group but did not achieve statistical significance (Figure 1D).

\section{KD Serum-Activated HCAEC Supernatant HMGB-1 and sRAGE Levels and HMGB-1 Receptor Expression}

Supernatant HMGB-1 levels (Figure 2A) and sRAGE levels (Figure 2B) in the $\mathrm{KD}$ controls were significantly higher than those in the VH (each $P<0.001)$ and sJIA $(P<0.001$ and $P=$ 0.046 , respectively) groups. HMGB-1 levels in the $\mathrm{KD}+\mathrm{PSL}$ group were significantly lower than those in $\mathrm{KD}$ control and KD+PBS (each $P<0.001$ ) (Figure 2C) groups, whereas sRAGE levels did not significantly differ between the groups (Figure 2D).

Basal expression levels of RAGE, TLR2, and TLR4 receptors were increased in KD serum-activated HCAECs compared to those in VH serum-activated HCAECs (Figure 2E). Of KD serum-activated HCAECs, RAGE expression in the KD+PSL group was significantly lower than that in the $\mathrm{KD}$ control and $\mathrm{KD}+\mathrm{PBS}$ groups (each $P=$ 0.007); however, TLR2 and TLR4 expression did not differ significantly between the groups (Figure 2F).

\section{Immunofluorescence Staining for HMGB-1 in Serum-Activated HCAECs}

Representative images revealed that serum activation of HCAECs induced HMGB-1 release from the nucleus. Compared with the VH group, KD control and KD+PBS HCAECs showed increased HMGB-1 staining in the cytoplasm or the extracellular space (Figures 3A, a-c). Conversely, compared with $\mathrm{KD}$ control and $\mathrm{KD}+\mathrm{PBS}$ HCAECs, KD+PSL HCAECs showed significant reduction in cytoplasmic and extracellular HMGB-1 (Figures 3A, d). Quantitative analysis of the fluorescence intensity of HMGB-1 released from nucleus of serum-activated HCAECs revealed significantly lower values for $\mathrm{KD}+\mathrm{PSL}$ than $\mathrm{KD}$ control and KD+PBS HCAECs (each $P<0.001$ ) (Figure 3B).

\section{Phosphorylation of Mitogen-Activated Protein Kinase and NF- $\mathrm{kB}$, and NLRP3 Inflammasome in Endothelial Cell Lysates From KD Serum-Activated HCAECs}

To determine the role of mitogen-activated protein kinase signaling, we evaluated the levels of ERK, pERK, JNK, pJNK, p38, and pp38 in serum-activated HCAEC lysates. The ERK: $\beta$-actin, JNK: $\beta$-actin, and $\mathrm{p} 38: \beta$-actin ratios did not significantly differ between $\mathrm{VH}$ and 
TABLE 2 | Patient characteristics between Kawasaki disease (KD) and systemic juvenile idiopathic arthritis (SJIA).

\begin{tabular}{|c|c|c|c|}
\hline Group & KD $(n=8)$ & sJIA (n = 4) & $P$ value \\
\hline Male, N (\%) & $5(62.5)$ & $2(50.0)$ & 0.692 \\
\hline Age at onset (years) & $1.6(0.5-2.9)$ & $7.8(4.6-13.3)$ & 0.011 \\
\hline Body weight (kg) & $10.8(7.8-13.0)$ & $17.7(13.0-25.1)$ & 0.061 \\
\hline White blood cell count $\left(\times 10^{3} / \mu \mathrm{L}\right)$ & $15.8(11.9-18.1)$ & $14.6(12.1-24.4)$ & 1.000 \\
\hline Neutrophil count $\left(\times 10^{3} / \mu \mathrm{L}\right)$ & $11.7(7.9-14.5)$ & $11.7(10.0-20.0)$ & 0.734 \\
\hline Platelet count $\left(\times 10^{4} / \mu \mathrm{L}\right)$ & $32.8(28.3-41.5)$ & $59.0(34.6-66.4)$ & 0.089 \\
\hline Aspartate aminotransferase (IU/L) & $45(26-288)$ & $29(23-33)$ & 0.202 \\
\hline Alanine aminotransferase (IU/L) & $56(10-336)$ & $14(10-21)$ & 0.348 \\
\hline Lactate dehydrogenase (IU/L) & 336 (273-394) & $353(283-494)$ & 0.610 \\
\hline Total protein (g/dL) & $6.6(6.4-7.0)$ & $7.7(7.3-7.8)$ & 0.006 \\
\hline Albumin (g/dL) & $3.4(3.2-3.7)$ & $3.3(3.1-3.5)$ & 0.330 \\
\hline Sodium (mEq/L) & $134(129-134)$ & $138(137-142)$ & 0.006 \\
\hline C-reactive protein (mg/dL) & $6.9(4.4-10.5)$ & $6.9(5.3-9.0)$ & 1.000 \\
\hline
\end{tabular}

Data are expressed as median values and interquartile range (25th, 75th percentile), or number (proportion, \%).

Bold values indicate statistically significance in patient characteristics between KD group and sJIA group.
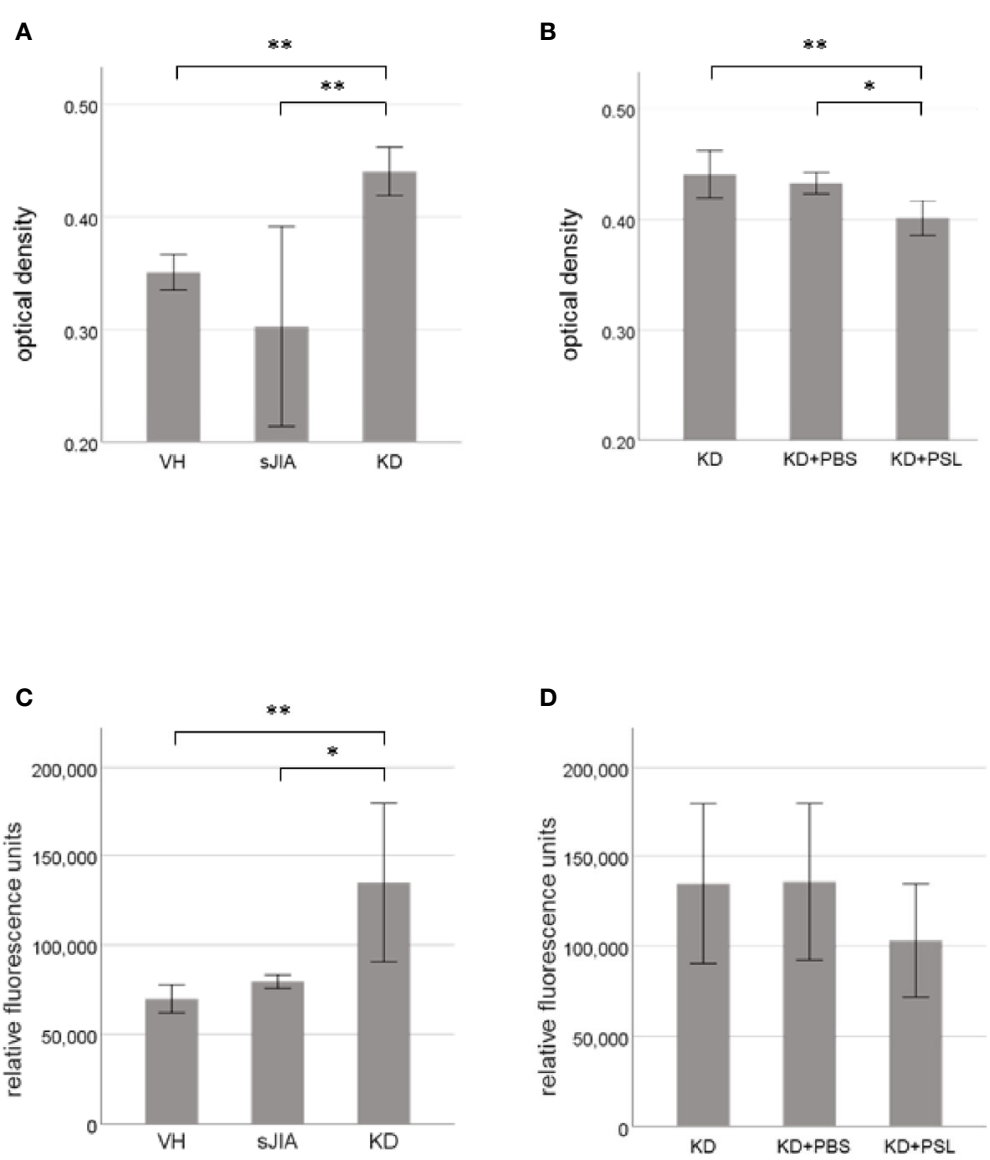

FIGURE 1 | Cell proliferation and cytotoxicity of serum-activated human coronary artery endothelial cells (HCAECs). MTT and cytotoxicity assay results for HCAECs stimulated with sera from healthy controls $(\mathrm{VH}, n=4)$ and patients with systemic juvenile idiopathic arthritis (sJIA, $n=4)$ or Kawasaki disease (KD; $n=8)$ for $24 \mathrm{~h}$ (A, C), and serum-activated HCAECs from patients with $\mathrm{KD}(n=8)$, and treated with PBS or prednisolone (PSL) for $24 \mathrm{~h}(\mathbf{B}, \mathbf{D}) .{ }^{\star} P<0.05$, ${ }^{\star \star} P<0.01[0 n e-$ way ANOVA followed by Bonferroni post-test (A-C) and Games-Howell post-test (D)].

KD groups; however, pERK: $\beta$-actin, $\mathrm{pJNK}: \beta$-actin and pp38: $\beta$-actin ratios in lysates from $\mathrm{KD}$ controls were significantly higher than those in VH lysates $(P<0.001)$. The ERK: $\beta$-actin, JNK: $\beta$-actin, and p38: $\beta$-actin ratios did not significantly differ between $\mathrm{KD}$ controls,
$\mathrm{KD}+\mathrm{PBS}$, and $\mathrm{KD}+\mathrm{PSL}$ groups, however, pERK: $\beta$-actin, pJNK: $\beta$ actin, and pp38: $\beta$-actin ratios were significantly lower in lysates from $\mathrm{KD}+\mathrm{PSL}$ groups than in lysates from the KD controls $(P=$ $0.004)$ and KD+PBS groups $(P=0.006)(p E R K: \beta$-actin; $P=0.004$ 


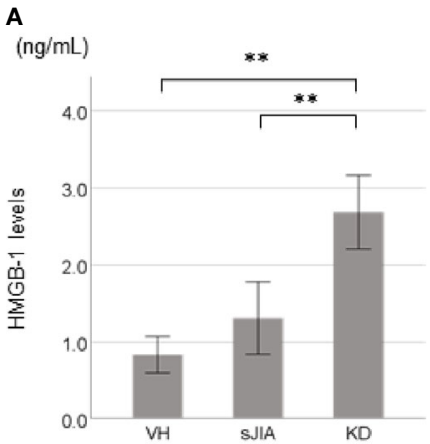

C

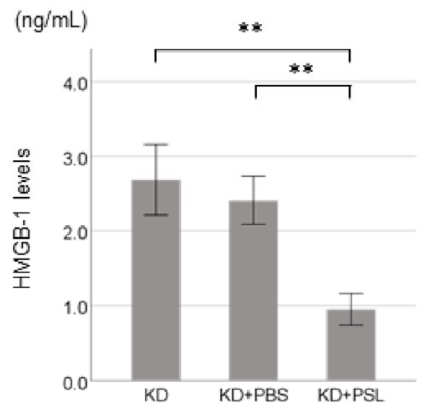

E

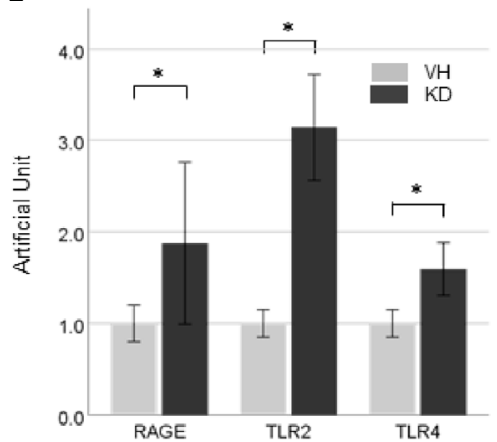

B

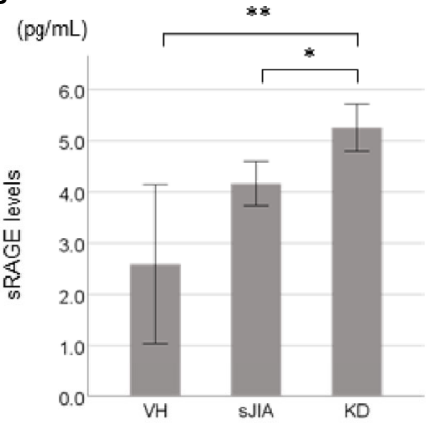

D

$(\mathrm{pg} / \mathrm{mL})$
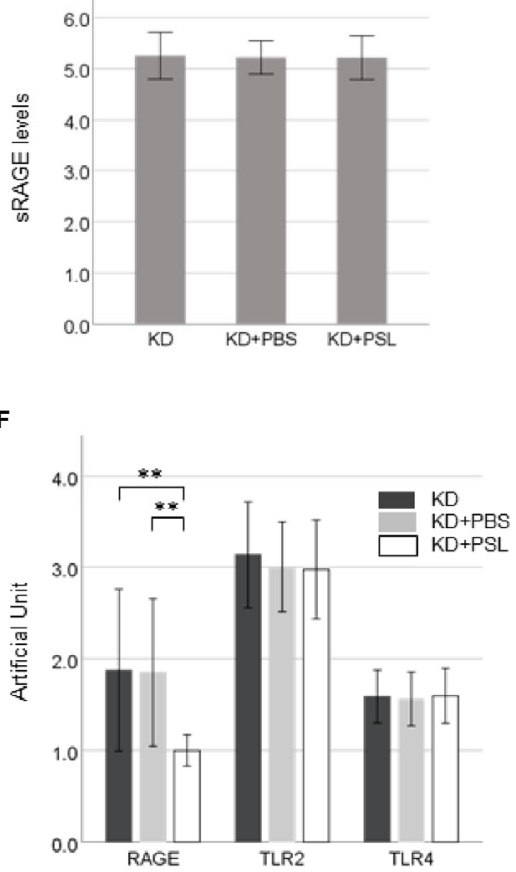

FIGURE 2 | HMGB-1 and SRAGE production in Kawasaki disease (KD)-serum-activated human coronary artery endothelial cells (HCAECs). HMGB-1 levels and sRAGE levels in supernatants from (A, B) healthy control $(\mathrm{VH}, n=4)$, systemic juvenile idiopathic arthritis (sJIA, $n=4)$, and KD ( $n=8)$ serum-activated HCAECs and (C, D) KD control, KD+PBS, and KD+prednisolone (PSL) HCAECs. Expression of HMGB-1 receptors in KD serum-activated HCAECs. Artificial Unit of RAGE, TLR2, and TLR4 receptors in (E) VH and KD serum-activated HCAECs, and (F) KD control, KD+PBS, and KD+PSL HCAECs. ${ }^{*} P<0.05$, ${ }^{\star *} P<0.01$ [one-way ANOVA followed by Bonferroni post-test (A, B, D, F) and Games-Howell post-test (C), Mann-Whitney U-tests (E)].

and $P=0.006$, pJNK: $\beta$-actin; $P<0.001$ and $P<0.001$, pp38: $\beta$-actin; $P=0.002$ and $P=0.004$, respectively) (Figure 4A).

The NLRP3:GAPDH and cleaved caspase 1:GAPDH ratios in the lysates from $\mathrm{KD}$ controls were higher than those in $\mathrm{VH}$ lysates ( $P=0.017$ and $P=0.018$, respectively), while the NLRP3: GAPDH and cleaved caspase 1:GAPDH ratios were lower in the lysates from $\mathrm{KD}+\mathrm{PSL}$ groups compared to the lysates from the $\mathrm{KD}$ controls and $\mathrm{KD}+\mathrm{PBS}$; no significant difference was observed between the groups (Figure 4B).

The total NF- $\mathrm{BB}$ p65:GAPDH ratio in cell lysates did not significantly differ between the nuclear and cytoplasmic fractions of the $\mathrm{VH}$ group, $\mathrm{KD}$ controls, $\mathrm{KD}+\mathrm{PBS}$ group, or $\mathrm{KD}+\mathrm{PSL}$ group. Conversely, the nuclear $\mathrm{p}-\mathrm{NF}-\kappa \mathrm{B}$ p65:GAPDH ratio in lysates from $\mathrm{KD}$ controls was significantly higher than those in lysates from the $\mathrm{VH}$ group $(P<0.001)$, whereas it was significantly reduced in the $\mathrm{KD}+\mathrm{PSL}$ group compared to that in $\mathrm{KD}$ control and $\mathrm{KD}+\mathrm{PBS}$ groups (each $P<0.001$ ) (Figure 4C).

\section{IL-1 $\beta$ and TNF- $\alpha$ Production in Endothelial Cell Lysates From KD Serum-Activated Hcaecs}

Both IL-1 $\beta$ and TNF- $\alpha$ levels in lysates of KD serum-activated HCAECs were significantly higher than those from the $\mathrm{VH}$ group (each $P<0.001$ ), whereas both were significantly lower 
A
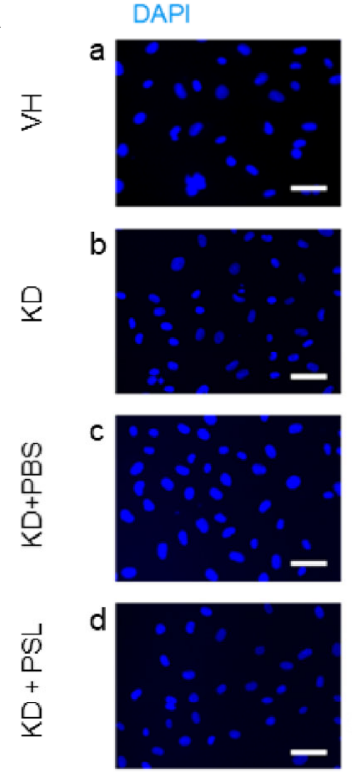

B
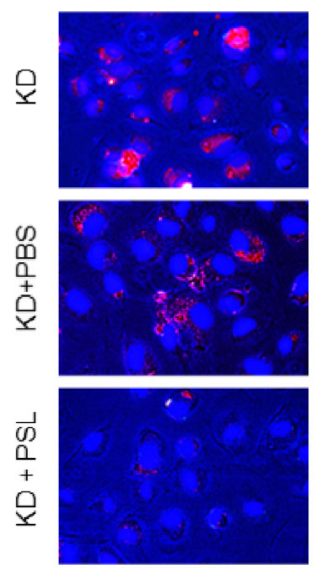

HMGB-1

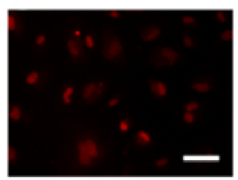

Merge
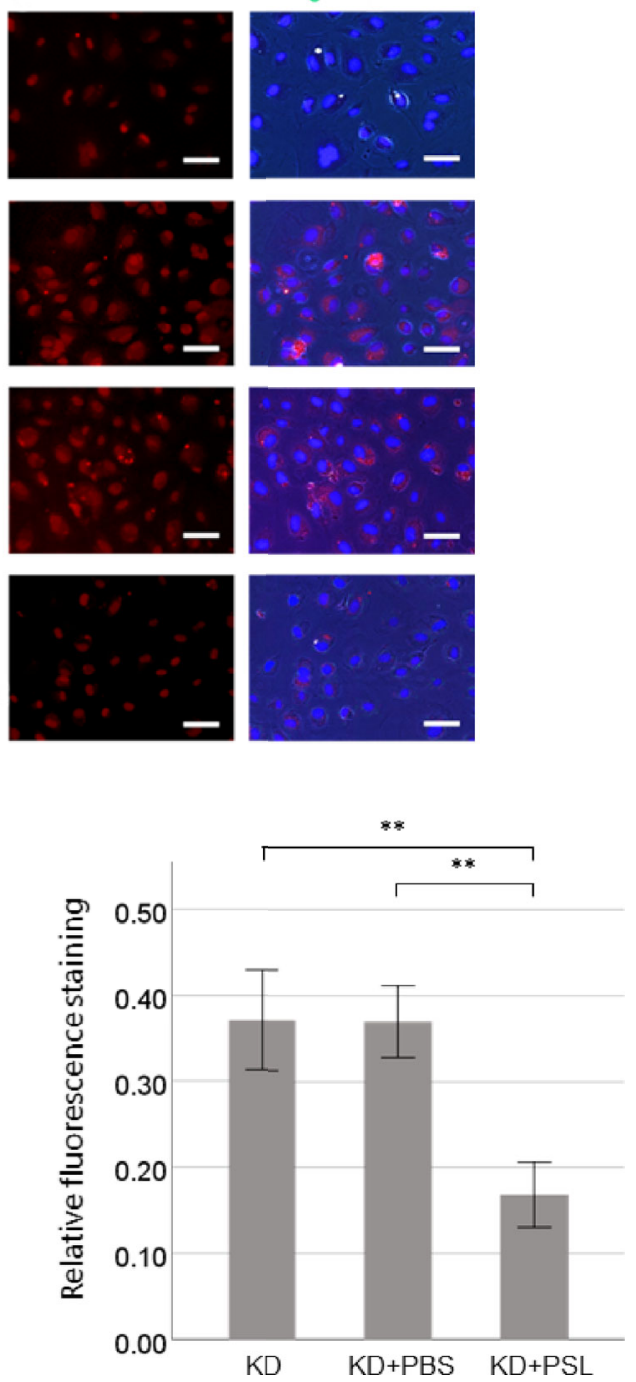

FIGURE 3 | Immunostaining of HMGB-1 in serum-activated human coronary artery endothelial cells (HCAECs) (magnification, x400). (A) Representative images of from healthy control $(\mathrm{VH})$, Kawasaki disease (KD), and KD serum-activated HCAECs treated with PBS and prednisolone (PSL) showing DNA (blue) labelled using DAPI, HMGB-1 (red) labelled using immunofluorescence staining, and merged images. Scale bar = $100 \mu \mathrm{m}$. (B) Relative fluorescence staining of extranuclear HMGB-1 from 100 cells, selected from KD serum-activated HCAECs alone and treated with PBS and PSL. Scale bar $=100 \mu$ m. ${ }^{* \star} P<0.01$ (one-way ANOVA followed by Bonferroni post-test).

in endothelial cell lysates from the $\mathrm{KD}+\mathrm{PSL}$ group than those from $\mathrm{KD}$ controls and $\mathrm{KD}+\mathrm{PBS}$ groups (IL- $1 \beta$, each $P<0.001$; TNF- $\alpha, P=0.001$, and $P=0.009$. respectively) (Figures 5A, B).

\section{DISCUSSION}

In this study, we demonstrated that serum obtained from patients with KD prior to IVIG treatment exhibited a cytotoxic effect on HCAECs compared to that from healthy controls and patients with sJIA, in addition to triggering extracellular release of HMGB-1, up-regulating NF- $\mathrm{KB}$-mediated inflammatory responses, and increasing IL- $1 \beta$ and TNF- $\alpha$ production. Although PSL treatment for KD serum-activated HCAECs did not show direct cytoprotective effects, it inhibited endothelial cell proliferation, HMGB-1 translocation, release, and downstream signaling and reduced IL- $1 \beta$ and TNF- $\alpha$ expression. These findings provide a new perspective regarding the antiinflammatory function of PSL during the acute phase of KD.

The vasculopathic process in the acute phase of $\mathrm{KD}$ involves necrotizing arteries progressively destroying the arterial wall from adventia to intima, particularly the coronary arteries (22, 23). Our results are in line with this pathological process in which the KD serum induced stronger cytotoxicity to coronary endothelial cells than sJIA serum, although there was no 
A
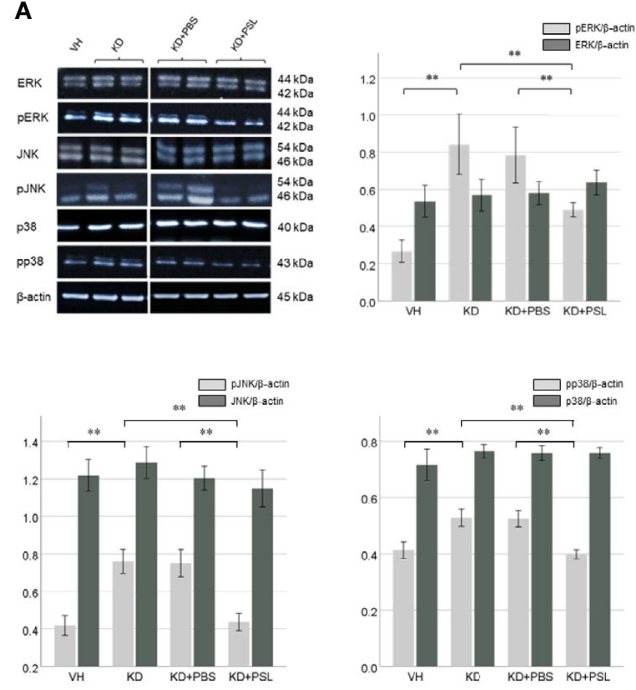

B

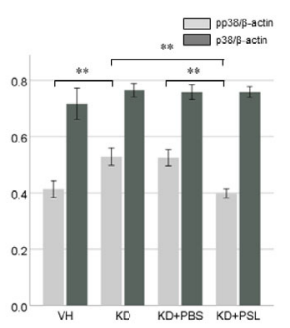

C
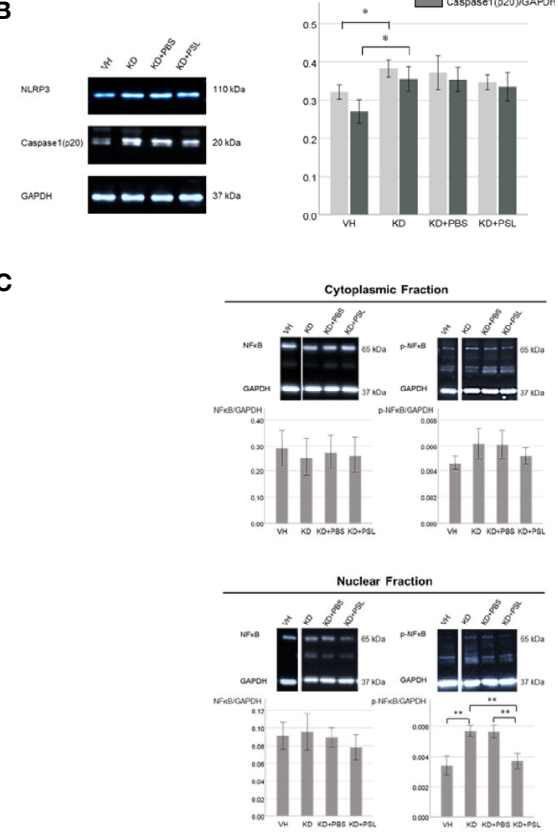

FIGURE 4 | ERK1/2, JNK, p38 and NF-KB levels in serum-activated human coronary artery endothelial cells (HCAECs). (A) Western blot analysis of ERK1/2, pERK1/2, JNK, pJNK, p38 and pp38 from healthy controls $(\mathrm{VH}, n=4)$, Kawasaki disease (KD, $n=8), \mathrm{KD}$ serum-activated HCAECs treated with PBS and prednisolone (PSL) (KD+PBS and KD+PSL, $n=8$, respectively). Bar graph shows immunoblotting results for the ERK1/2: $\beta$-actin, pERK 1/2: $\beta$-actin, JNK: $\beta$-actin, pJNK: $\beta$-actin, p38: $\beta$-actin and pp38: $\beta$-actin ratio ${ }^{* *} P<0.01$ (one-way ANOVA followed by the Bonferroni, post-test). (B) NLRP3 and Caspase-1(p20) in serumactivated HCAECs from healthy controls $(\mathrm{VH}, n=4), \mathrm{KD}(n=8)$, and $\mathrm{KD}$ serum-activated HCAECs treated with PBS and PSL (KD+PBS and KD+PSL, $n=8$, respectively). Bar graphs show immunoblotting results for NLRP3:GAPDH and Caspase-1(p20):GAPDH ratio in HCAEC lysates. ${ }^{*} P<0.05$ (Kruskal-Wallis test

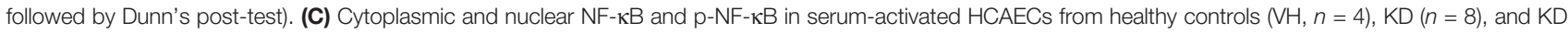
serum-activated HCAECs treated with PBS and PSL (KD+PBS and KD+PSL, $n=8$, respectively). Bar graphs show immunoblotting results for NF-KB:GAPDH and p-NF-kB:GAPDH ratio in HCAEC lysates. ${ }^{* *} P<0.01$ (Kruskal-Wallis test followed by Dunn's post-test).

difference in patients' laboratory data between $\mathrm{KD}$ and sJIA. Since KD serum also up-regulated DAMP receptors, such as RAGE and TLRs, and induced subsequent intracellular activation of downstream ERK1/2, JNK, p38, and NF- $\mathrm{KB}$ signaling pathways in HCAECs, endothelial cell damage consequent to pathogenic proteins in $\mathrm{KD}$ serum may thus underlie several of the pathological features of $\mathrm{KD}$ and the effects observed during the early stages of disease progression. Specifically, during cytotoxic response to KD serum, HMGB-1 translocated from the nucleus to the extracellular space, where it may function as a DAMP or alarmin to stimulate the innate immune system and mediate inflammation in accordance with its role in the pathogenesis of delayed inflammatory responses and organ dysfunction (24).

Moreover, extracellular HMGB-1 can interact with RAGE or TLRs on the surface of inflammatory endothelial cells. HMGB-1 functional interaction with receptors activates inflammationassociated pathways and triggers a cascade of proinflammatory cytokines, including ILs, TNF- $\alpha$, and macrophage inflammatory protein- $1 \alpha$ and $-1 \beta$, thereby forming a self-reinforcing inflammatory cycle $(25,26)$. As serum levels of HMGB-1 and S100 are elevated during the acute $\mathrm{KD}$ phase $(7,15-17)$ and RAGE activation results in up-regulated proinflammatory cytokine expression in patients with $\mathrm{KD}(18)$, these processes may stimulate granulocytes or endothelial cells to secrete DAMPs, thereby establishing a self-amplifying positive feedback loop. However, sRAGE, a truncated soluble form of the receptor, acts as a decoy and prevents the RAGE activationmediated inflammatory response (27). Consistent with our results, Wittkowski et al. (28) found that sRAGE levels in acute KD were significantly lower than those post-IVIG or in the subacute phase, suggesting the potential anti-inflammatory effect of sRAGE on inflammatory vascular disorders. Additionally, subsequent activation of HMGB-1/RAGE-specific downstream signaling pathways and increased levels of IL-1 $\beta$ or TNF- $\alpha$ constitute parallel or consecutive events in response to increased HMGB-1 levels. The HMGB-1/RAGE signaling pathway in endothelial cells also induces cathepsin B activation, subsequently inducing canonical pyroptosis via NLRP3 inflammasomes in KD vasculitis (18). Therefore, our study also supports the hypothesis that extracellular HMGB-1 is possibly up-regulated to act as a functional cytokine influencing inflammation and the innate immune response, and that it may thus contribute to the pathogenesis of $\operatorname{KD}$ vasculitis $(29,30)$.

We further revealed that PSL treatment of KD serumactivated HCAECs inhibited extracellular HMGB-1 release, reduced $R A G E$ expression, and inhibited NF- $\mathrm{KB}-$ mediated inflammatory responses, in addition to reducing IL- $1 \beta$ and 
A

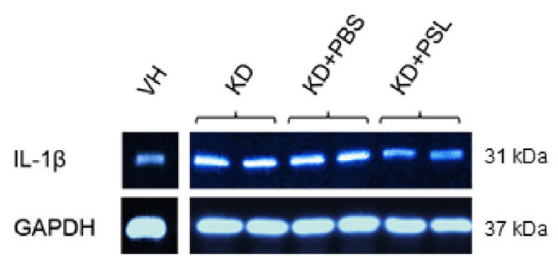

B

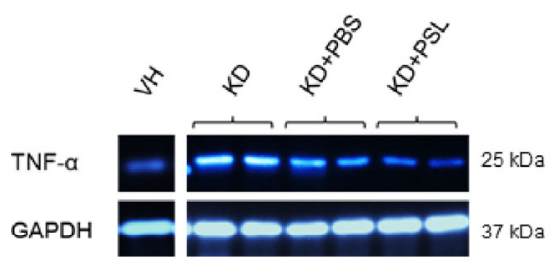

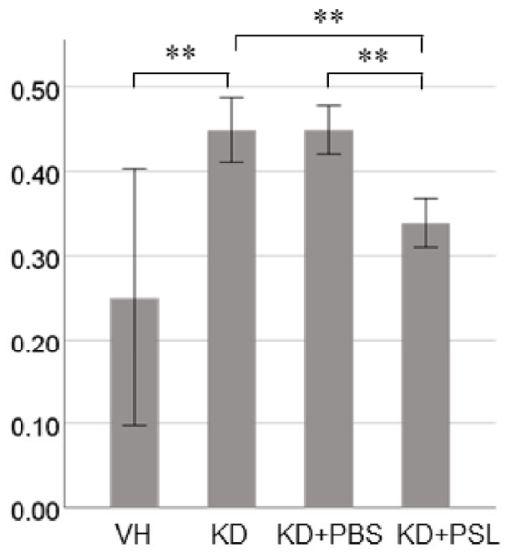

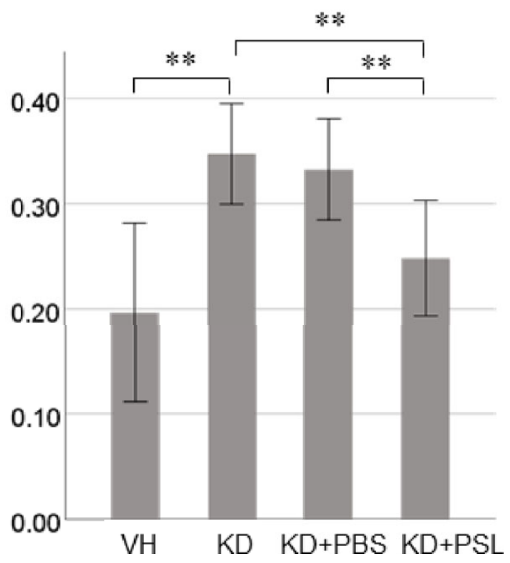

FIGURE 5 | Cytokine production in serum-activated human coronary artery endothelial cell (HCAEC) lysates. Western blot analysis and quantitation of (A) IL-1 $\beta$ and (B) TNF- $\alpha$ production in serum-activated HCAEC lysates from healthy controls (VH) and Kawasaki disease (KD), and KD serum-activated HCAECs treated with PBS and prednisolone (PSL) (8 independent experiments). GAPDH was used as an internal standard. ${ }^{\star \star} P<0.01$ (one-way ANOVA followed by Bonferroni post-test).

TNF- $\alpha$ production. Conversely, PSL treatment did not show cytoprotective effects or sRAGE up-regulation in KD-serumactivated HCAECs, supporting that the effects of PSL treatment might occur by directly inhibiting extracellular HMGB-1 release. In general, the anti-inflammatory effects of glucocorticoids are attributable to the transcriptional effects of glucocorticoidreceptor agonism, which alters the transcription of numerous genes both positively and negatively by targeting specific cell populations to combat the immune system hyperactivation or systemic infections $(10,31-33)$. Glucocorticoid treatment inhibits the expression of proinflammatory genes, including $\mathrm{NF}-\kappa \mathrm{B}$ and activator protein 1 (10); therefore, the observed PSL-induced inhibition of HMGB-1 release might occur via NF- $\kappa \mathrm{B}$ signaling.

Another possible mechanism is that glucocorticoids inhibit TNF- $\alpha$ synthesis in activated monocytes/macrophages, a process at least partially involved in the anti-inflammatory effects of TNF$\alpha$-induced HMGB-1 secretion from endothelial cells (30). Therefore, PSL treatment of KD serum-activated HCAECs likely has an upstream regulatory component and potentially inhibits the HMGB-1 signaling pathway. CD14 is important in efficient HMGB-1-dependent TLR activation $(34,35)$, whereas RAGE offers a different transduction pathway in providing a transport route for HMGB-1 and its partner molecule complexes by endocytosis to the endolysosomal component (36). Rather than being degraded in the lysosomes, HMGB-1 transported molecules then leak out from the permeabilized lysosomes into the cytosol to reach and activate cognate cytoplasmic receptors, thereby causing inflammation (36). In the present study, we could not confirm a functional role for the HMGB-1/TLRs signaling pathway and HMGB-1 induced NLRP3 inflammasome activation through the serum stimulation experiments using cultured HCAECs owing to the lack of lymphocytes and macrophages. However, our findings suggest that PSL application during acute $\mathrm{KD}$ has a distinct potential to also ameliorate HMGB-1/RAGE-mediated inflammatory responses in $\mathrm{KD}$ vasculitis, which is borne out by the efficacy of glucocorticoids at reducing the incidence of CAA and the number of IVIG non-responders in KD (4-6). 
Nevertheless, there were several limitations in this study with respect to the effects of PSL treatment in $\mathrm{KD}$ vasculitis that remain unaddressed. Serum samples from patients with KD used in this study were limited; hence, we did not measure cytokine and inflammatory markers other than those from routine blood testing. Glucocorticoids affect virtually all immune cells and their precise effects depend on the differentiation and activation state of the cell, making interpretation of in vivo effects in specific populations difficult. Glucocorticoids inhibit neutrophildependent endothelial cell injury (37) or platelet-neutrophil aggregate formation (38), thereby reducing cytokine-induced adhesion and inhibiting amplified reciprocal vascular inflammatory activation. However, we could not examine the contribution of neutrophil-, monocyte-, and platelet-dependent endothelial cell activation since our serum-stimulation experiments were performed using cultured coronary endothelial cells. Given the differences in glucocorticoid-receptor levels between endothelial cells and various vascular beds, the relative proportion of specific glucocorticoid-receptor isoforms in tissues and cells may influence their responses to glucocorticoid treatment (39-41). IVIG is the standard and most effective treatment for $\mathrm{KD}$, however, the present study focused on the mechanism of action of corticosteroids in KD; and hence no examination or analysis of the effects of IVIG was conducted in this study.

In conclusion, our findings suggest that extracellular HMGB1 is potentially up-regulated to act as a functional cytokine with roles in both inflammation and the innate immune response, thereby mediating KD pathogenesis. Treatment with PSL during the acute phase of KD ameliorates HMGB-1/RAGE-mediated inflammatory responses and reduces IL-1 $\beta$ and TNF- $\alpha$ production. Inhibiting extracellular HMGB-1 may also inhibit the over-activated innate immune system, thus offering potential relief from or prevention of severe $\mathrm{KD}$ vasculitis.

\section{DATA AVAILABILITY STATEMENT}

The datasets presented in this study can be found in online repositories. The names of the repositories and accession numbers can be found in the article/Supplementary Material.

\section{REFERENCES}

1. Kawasaki T, Kosaki F, Okawa S, Shigematsu I, Yanagawa H. A New Infantile Acute Febrile Mucocutaneus Lymph Node Syndrome (MLNS) Prevailing in Japan. Pediatrics (1974) 54:271-6.

2. Wallace CA, French JW, Kahn SJ, Sherry DD. Initial Intravenous Gammaglobulin Treatment Failure in Kawasaki Disease. Pediatrics (2000) 105:E78. doi: 10.1542/peds.105.6.e78

3. Burns JC, Capparelli EV, Brown JA, Newburger JW, Glode MP. Intravenous Gamma-Globulin Treatment and Retreatment in Kawasaki Disease. Pediatr Infect Dis J (1998) 17:1144-8. doi: 10.1097/00006454-199812000-00009

4. Kobayashi T, Saji T, Otani T, Takeuchi K, Nakamura T, Arakawa H, et al. Efficacy of Immunoglobulin Plus Prednisolone for Prevention of Coronary Artery Abnormalities in Severe Kawasaki Disease (RAISE Study): A Randomised, Open-Label, Blinded-Endpoints Trial. Lancet (2012) 379:1613-20. doi: 10.1016/S0140-6736(11)61930-2

\section{ETHICS STATEMENT}

The studies involving human participants were reviewed and approved by Kagoshima University and Kagoshima City Hospital Ethics Committee. Written informed consent to participate in this study was provided by the participants' legal guardian/next of kin. Written informed consent was obtained from the individual(s), and minor(s)' legal guardian/next of kin, for the publication of any potentially identifiable images or data included in this article.

\section{AUTHOR CONTRIBUTIONS}

$\mathrm{KU}, \mathrm{YN}$ and YK conceived and designed the experiments. KU and YM performed the experiments. KU and YN analyzed the data and designed the figures. KU performed the statistical analysis and wrote the first draft of the manuscript. All authors contributed to the article and approved the submitted version.

\section{FUNDING}

This work was supported financially by a Japan Society for the Promotion of Science (JSPS) KAKENHI Grant Numbers JP16K19657 and JP19K08302.

\section{ACKNOWLEDGMENTS}

We wish to express our appreciation to the patients and their families for their commitment to this project. We thank the laboratory technicians Junko Hirose, Chizuru Tamari, and Emiko Miyahara for their help with our experiments.

\section{SUPPLEMENTARY MATERIAL}

The Supplementary Material for this article can be found online at: https://www.frontiersin.org/articles/10.3389/fimmu.2021. 640315/full\#supplementary-material
5. Newburger JW, Sleeper LA, McCrindle BW, Minich LL, Gersony W, Vetter VL, et al. Randomized Trial of Pulsed Corticosteroid Therapy for Primary Treatment of Kawasaki Disease. N Engl J Med (2007) 356:663-75. doi: 10.1056/NEJMoa061235

6. Ogata S, Ogihara Y, Honda T, Kon S, Akiyama K, Ishii M. Corticosteroid Pulse Combination Therapy for Refractory Kawasaki Disease: A Randomized Trial. Pediatrics (2012) 129:e17-23. doi: 10.1542/peds.2011-0148

7. Hara T, Nakashima Y, Sakai Y, Nishio H, Motomura Y, Yamasaki S. Kawasaki Disease: A Matter of Innate Immunity. Clin Exp Immunol (2016) 186:134-43. doi: $10.1111 /$ cei.12832

8. Tang D, Kang R, Livesey KM, Cheh CW, Farkas A, Loughran P, et al. Endogenous HMGB1 Regulates Autophagy. J Cell Biol (2010) 190:881-92. doi: $10.1083 /$ jcb.200911078

9. Tang D, Kang R, Coyne CB, Zeh HJ, Lotze MT. Pamps and DAMPs: Signal 0s That Spur Autophagy and Immunity. Immunol Rev (2012) 249:158-75. doi: 10.1111/j.1600-065X.2012.01146.x 
10. Andersson U, Tracey KJ. HMGB1 is a Therapeutic Target for Sterile Inflammation and Infection. Annu Rev Immunol (2011) 29:139-62. doi: 10.1146/annurev-immunol-030409-101323

11. Kohno T, Anzai T, Naito K, Miyasho T, Okamoto M, Yokota H, et al. Role of High-Mobility Group Box 1 Protein in Post-Infarction Healing Process and Left Ventricular Remodelling. Cardiovasc Res (2009) 81:565-73. doi: 10.1093/ $\mathrm{cvr} / \mathrm{cvn} 291$

12. Liu T, Zhang DY, Zhou YH, Han QF, Wang LH, Wu L, et al. Increased Serum HMGB1 Level may Predict the Fatal Outcomes in Patients With Chronic Heart Failure. Int J Cardiol (2015) 184:318-20. doi: 10.1016/j.ijcard. 2015.02.088

13. Mehra VC, Ramgolam VS, Bender JR. Cytokines and Cardiovascular Disease. J Leukoc Biol (2005) 78:805-18. doi: 10.1189/jlb.0405182

14. Paoletti R, Gotto AMJr, Hajjar DP. Inflammation in Atherosclerosis and Implications for Therapy. Circulation (2004) 109:III20-6. doi: 10.1161/ 01.CIR.0000131514.71167.2e

15. Ebihara T, Endo R, Kikuta H, Ishiguro N, Ma X, Shimazu M, et al. Differential Gene Expression of S100 Protein Family in Leukocytes From Patients With Kawasaki Disease. Eur J Pediatr (2006) 164:427-31. doi: 10.1007/s00431-0051664-5

16. Hoshina T, Kusuhara K, Ikeda K, Mizuno Y, Saito M, Hara T. High Mobility Group Box 1 (HMGB1) and Macrophage Migration Inhibitory Factor (MIF) in Kawasaki Disease. Scand J Rheumatol (2008) 37:445-9. doi: 10.1080/ 03009740802144143

17. Eguchi T, Nomura Y, Hashiguchi T, Masuda K, Arata M, Hazeki D, et al. An Elevated Value of High Mobility Group Box 1 is a Potential Marker for Poor Response to High-Dose of Intravenous Immunoglobulin Treatment in Patients With Kawasaki Syndrome. Pediatr Infect Dis J (2009) 28:339-41. doi: 10.1097/INF.0b013e31818ffe60

18. Jia C, Zhang J, Chen H, Zhuge Y, Chen H, Qian F, et al. Endothelial Cell Pyroptosis Plays an Important Role in Kawasaki Disease Via HMGB1/RAGE/ Cathespin B Signaling Pathway and NLRP3 Inflammasome Activation. Cell Death Dis (2019) 10:778. doi: 10.1038/s41419-019-2021-3

19. Ayusawa M, Sonobe T, Uemura S, Ogawa S, Nakamura Y, Kiyosawa N, et al. Revision of Diagnostic Guidelines for Kawasaki Disease (the 5th Revised Edition). Pediatr Int (2005) 47:232-34. doi: 10.1111/j.1442-200x.2005.02033.x

20. Yoon JS, Kim HH, Han JW, Lee Y, Lee JS. Effects of Intravenous Immunoglobulin and Methylprednisolone on Human Umbilical Endothelial Cells In Vitro. Immunobiology (2006) 211:351-57. doi: 10.1016/ j.imbio.2006.02.003

21. Schneider CA, Rasband WS, Eliceiri KW. Nih Image to ImageJ: 25 Years of Image Analysis. Nat Methods (2012) 9:671-5. doi: 10.1038/nmeth.2089

22. Shulman ST, Rowley AH. Kawasaki Disease: Insights Into Pathogenesis and Approaches to Treatment. Nat Rev Rheumatol (2015) 11:475-82. doi: 10.1038/nrrheum.2015.54

23. Orenstein JM, Shulman ST, Fox LM, Baker SC, Takahashi M, Bhatti TR, et al. Three Linked Vasculopathic Processes Characterize Kawasaki Disease: A Light and Transmission Electron Microscopic Study. PLoS One (2012) 7: e38998. doi: 10.1371/journal.pone.0038998

24. Bangert A, Andrassy M, Müller AM, Bockstahler M, Fischer A, Volz CH, et al. Critical Role of RAGE and HMGB1 in Inflammatory Heart Disease. Proc Natl Acad Sci U S A (2016) 113:E155-64. doi: 10.1073/pnas.1522288113

25. Scaffidi P, Misteli T, Bianchi ME. Release of Chromatin Protein HMGB1 by Necrotic Cells Triggers Inflammation. Nature (2002) 418:191-5. doi: 10.1038/ nature 00858

26. Yan XX, Lu L, Peng WH, Wang LJ, Zhang Q, Zhang RY, et al. Increased Serum HMGB1 Level is Associated With Coronary Artery Disease in Nondiabetic and Type 2 Diabetic Patients. Atherosclerosis (2009) 205:544-8. doi: 10.1016/j.atherosclerosis.2008.12.016

27. Lanati N, Emanuele E, Brondino N, Geroldi D. Soluble RAGE-modulating Drugs: State-of-the-Art and Future Perspectives for Targeting Vascular
Inflammation. Curr Vasc Pharmacol (2010) 8:86-92. doi: 10.2174/ 157016110790226642

28. Wittkowski H, Hirono K, Ichida F, Vogl T, Ye F, Yanlin X, et al. Acute Kawasaki Disease is Associated With Reverse Regulation of Soluble Receptor for Advance Glycation End Products and its Proinflammatory Ligand S100A12. Arthritis Rheum (2007) 56:4174-81. doi: 10.1002/art.23042

29. Erlandsson Harris H, Andersson U. Mini-Review: The Nuclear Protein HMGB1 as a Proinflammatory Mediator. Eur J Immunol (2004) 34:150312. doi: $10.1002 /$ eji.200424916

30. Yang WS, Han NJ, Kim JJ, Lee MJ, Park SK. Tnf- $\alpha$ Activates High-Mobility Group Box 1-Toll-like Receptor 4 Signaling Pathway in Human Aortic Endothelial Cells. Cell Physiol Biochem (2016) 38:2139-51. doi: 10.1159/ 000445570

31. Adcock IM. Molecular Mechanisms of Glucocorticosteroid Actions. Pulm Pharmacol Ther (2000) 13:115-26. doi: 10.1006/pupt.2000.0243

32. Miura M. Role of Glucocorticoids in Kawasaki Disease. Int J Rheum Dis (2018) 21:70-5. doi: 10.1111/1756-185X.13209

33. Busillo JM, Cidlowski JA. The Five Rs of Glucocorticoid Action During Inflammation: Ready, Reinforce, Repress, Resolve, and Restore. Trends Endocrinol Metab (2013) 24:109-19. doi: 10.1016/j.tem.2012.11.005

34. Kim S, Kim SY, Pribis JP, Lotze M, Mollen KP, Shapiro R, et al. Signaling of High Mobility Group Box 1 (HMGB1) Through Toll-Like Receptor 4 in Macrophages Requires CD14. Mol Med (2013) 19:88-98. doi: 10.2119/ molmed.2012.00306

35. Armaroli G, Verweyen E, Pretzer C, Kessel K, Hirono K, Ichida F, et al. Monocyte-Derived interleukin-1beta as the Driver of S100A12-induced Sterile Inflammatory Activation of Human Coronary Artery Endothelial Cells: Implications for the Pathogenesis of Kawasaki Disease. Arthritis Rheum (2019) 71:792-804. doi: 10.1002/art.40784

36. Deng M, Tang Y, Li W, Wang X, Zhang R, Zhang X, et al. The Endotoxin Delivery Protein HMGB1 Mediates caspase-11-dependent Lethality in Sepsis. Immunity (2018) 49:740-53.e7. doi: 10.1016/j.immuni.2018.08.016

37. Heimbürger M, Lärfars G, Bratt J. Prednisolone Inhibits Cytokine-Induced Adhesive and Cytotoxic Interactions Between Endothelial Cells and Neutrophils In Vitro. Clin Exp Immunol (2000) 119:441-8. doi: 10.1046/ j.1365-2249.2000.01165.x

38. Ueno K, Nomura Y, Morita Y, Eguchi T, Masuda K, Kawano Y. Circulating Platelet-Neutrophil Aggregates Play a Significant Role in Kawasaki Disease. Circ J (2015) 79:1349-56. doi: 10.1253/circj.CJ-14-1323

39. Coutinho AE, Chapman KE. The Anti-Inflammatory and Immunosuppressive Effects of Glucocorticoids, Recent Developments and Mechanic Insights. Mol Cell Endocrinol (2011) 335:2-13. doi: 10.1016/ j.mce.2010.04.005

40. Koenen P, Barczyk K, Wolf M, Roth J, Viemann D. Endothelial Cells Present an Innate Resistance to Glucocorticoid Treatment: Implications for Therapy of Primary Vasculitis. Ann Rheum Dis (2012) 71:729-36. doi: 10.1136/ annrheumdis-2011-200530

41. Lu NZ, Collins JB, Grissom SF, Cidlowski JA. Selective Regulation of Bone Cell Apoptosis by Translational Isoforms of the Glucocorticoid Receptor. Mol Cell Biol (2007) 27:7143-60. doi: 10.1128/MCB.00253-07

Conflict of Interest: The authors declare that the research was conducted in the absence of any commercial or financial relationships that could be construed as a potential conflict of interest.

Copyright () 2021 Ueno, Nomura, Morita and Kawano. This is an open-access article distributed under the terms of the Creative Commons Attribution License (CC BY). The use, distribution or reproduction in other forums is permitted, provided the original author(s) and the copyright owner(s) are credited and that the original publication in this journal is cited, in accordance with accepted academic practice. No use, distribution or reproduction is permitted which does not comply with these terms. 